\title{
Quantitative Analysis of Metastasis Brain Tumor and its Area Estimation in MR Images
}

\author{
K. Vidyasagar \\ Department of ECE, SSIT, \\ Sattupally
}

\author{
A. Bhujangarao \\ Deaprtment of Instrument \\ Technology, Andhra University
}

\author{
T. Madhu \\ Swarnandhra Institute of \\ Engineering \& Technology, \\ Narasapur
}

\begin{abstract}
Metastasis brain tumor develops multiple tumors at asymmetrical location of the human brain. MRI Imaging is one of the prudent mechanisms to extract the tumor regions and to map the brain for diagnosing. For the better diagnosis, one must detect the tumor accurately and need to calculate the area and volume of the tumor exactly. Here in this letter, we proposed a novel resolution enhancement technique to improve the quality of MR brain image and optimized hybrid clustering with region split and merge algorithm to detect the tumor cells from the original MR images and to estimate the tumors from different locations. Simulation results show that the proposed algorithm has performed superior to conventional clustering algorithms such as Fuzzy C-means (FCM), K- Means and even optimized pillar algorithm.
\end{abstract}

\section{Keywords}

Metastasis brain tumor, DWT, SWT, Interpolation, Image Segmentation, FCM, K-means, Optimized pillar algorithm

\section{INTRODUCTION}

The cells in a human brain progress in a controlled manner. The old and flawed cells recovered with new cells. The cell division grows in capricious style and form as aberrant tissue labeled as Tumor. Tumors are classified as primary and secondary tumors. The origins of primary tumors develop with in the brain. When cells split from the primary tumor and shipment through the blood system to another fragment of the body called Secondary tumors or metastases. Secondary tumors are habitually evolving multiple tumors. Secondary brain tumors arise in the lung, breast, kidney, or from melanomas in the skin. Metastatic brain tumors can develop expeditiously, congregate or spoiling nearby brain tissue. Consistently, a patient may have multiple metastatic tumors in distinct areas of the brain. It is well known by all of us that the brain tumor is inherently serious and life-threatening because of its character in the limited space of the intracranial cavity (space formed inside the skull). Most Researchers from the developed countries has proven that number of people who have brain tumors were died due to the fact of inaccurate tumor detection and estimation of its size. To produce the complete brain images, we will use CT scan or MRI, which is directed into intracranial cavity of brain. After then, for detection \& diagnosis of brain tumor, the scanned brain image will be visually examined by the physician. However this method of detection resists the accurate determination of stage $\&$ size of tumor. To avoid the conventional drawbacks such as low resolution images, inaccurate detection of tumor and its size, this thesis work proposed a computer aided method for MR brain image segmentation [1] for detecting the tumor cells from different locations of image i.e., from metastasis brain image.

In the proposed approach the low resolution MR image is enhanced by applying the well known transformation techniques named as discrete wavelet transform (DWT) [2], [3], [7] and stationary wavelet transform (SWT) [4], [5], [6] to resolve the problem of low resolution and to increase the contrast and sharpness of the image. Then after, we had applied proposed optimized hybrid clustering with region split and merge algorithm to detect the tumor cells from the high resolute MR image. This process includes a new and novel mechanism for clustering the elements of high-resolution images in order to improve the precision and reduce computation time.

\section{RELEVANT WORK}

From the past decades many researchers have developed the MR brain image segmentation algorithms by applying the segmentation algorithms and clustering techniques to detect the tumor cells from the MR images [1]. The author in [2] proposed An Automated 3D Segmented and DWT Enhanced Model for Brain MRI. In this context, the author used DWT to enhance the MR image from its low resolution image and then by using manual segmentation is will be segmented automatically. But, we know that the manual segmentation will consume more time, which in results the increment in computational time. This will help us that this approach is not suitable for MR image segmentation and by considering the results progression, significant number of miss detected and false detected pixels causes to produce in accurate tumor area and also will not detect the tumor cells accurately. The authors from [3], [4], [5], [6] and [7] had proposed image enhancement techniques using transformation approaches such as DWT and SWT decomposition. In [3] and [4], author developed a discrete wavelet based enhancement techniques to enhance the low resolution MR image. However, DWT is a decimated approach, in which the input image will be decimated with a factor of 2 i.e., the size of DWT sub bands will be reduced to half of the input image. Hence, it is not suitable for lossless enhancement. To overcome this, authors in [5], [6] and [7] proposed an extension version for DWT which does not use decimation factor of 2 named as stationary wavelet transform (SWT), by using the combination of both DWT and SWT original low resoluted image will be enhanced more effectively and will produce the better performance than the DWT approaches. However, due to the lack of number of original pixels all of them were fail to produce a high resoluted image without losing its original content information from the MR images. Manisha et. al. in [8] proposed an improved watershed segmentation algorithm, which provides better results than the manually segmented algorithms but it includes few drawbacks like oversegmentation and sensitivity to false edges. Recent years, fazel in [9] proposed a fuzzy expert approach for segmenting the tumor cells from the MR brain images. However, that the fuzzy approach has produced good segmented results but it suffers from determining the membership function, which is 
used to cluster the similar pixels in MR image. Fuzzy will be worked with allocation of membership function to the pixels based on the initial centroids selected from the pixels of input image, which is to be segmented. Specifically, determining the number of the cluster is a considerable limitation with FCM. Since the regions are spatially discontinuous grey level similarity is only verified. Considering the experimental study FCM is converging to local minima of the squared error criterion [9]. Then after, Mohammed et. al. in [10] proposed an efficient brain tumor detection scheme based on the combination of spatial information with fuzzy c-means which overcomes the drawback found in [9], but it takes much time to segment the tumor and will suffer from false edges. To overcome, the drawbacks of manually segmented, watershed and FCM [7], [8], [9], and [10] clustering algorithms, Mary Praveena in [11] proposed a fusion based image segmentation using k-means clustering, which is an extension to the above mentioned algorithms and will provide the best results within less computational time. Later years, there are so many algorithms such as histogram based approach, anisotropic diffusion and FCM have been merged with the k-means and given the best performance over conventional techniques [12], [13], [14] and [15]. However, this k-Means is limited to produce only hyper spherical clusters. It depends on initial centroids. To update the new centroids the mean of the pixel values of the respective clusters need to be estimated. The floating values obtained in some iteration are not favorable. Significantly, positive integers or scalars are required to replace the new centroids. Hence with K-means algorithm the optimal solution is difficult to achieve. In order to overcome the drawbacks of k-means, Barakbah et. al. in [16] proposed a pillar algorithm to solve the initial centroid designation problem, by considering the pixel maximization i.e., select maximum pixel value for centroid. To improve the performance of segmentation and detecting the accurate tumor cell from the MR image, pillar algorithm will be merged with k-means clustering, which is proposed in [17] and also proposed feature extraction with approximate reasoning to calculate the area of tumor based on the number of white pixels in the segmented MR brain image. The number of clusters will be selected optimally and also the centroid updation for number of iteration will also be solved in pillar $\mathrm{k}$-means by considering the maximum pixel value instead of calculating the mean of group of clustered pixel values [17]. All the above mentioned algorithms have been developed and tested for primary tumor images, in which the MR images have only single tumor cell, and will not mention the medical parameters such as accuracy, sensitivity of the tumor. Here in this letter, we proposed super resolution enhancement using DWT and SWT with Bi-cubic interpolation to enhance the MR image quality and optimized hybrid clustering with region split and merge algorithm to detect the exact tumor location and to calculate the individual areas of multiple tumors from the MR images.

\section{PROPOSED METHADOLOGY}

\subsection{Super Resolution Image Enhancement}

Discrete wavelet transform and Stationary wavelet transform are used to resolute the Low resolution MR input image. The information loss on its edges i.e., high frequency components may arise by applying the interpolation for image intensification. Safeguard the edges is imperative. DWT has been hired to safeguard the high frequency components of the image. In-order to obtain the redundancy and shift invariance the discrete wavelet transform coefficients are essentially interpolated. Hence DWT is used to disintegrate the MRI Image into sub band images. The high frequency components of the image labeled as sub bands LH, HL, HH. For interpolating data points on a two dimensional regular grid, $\mathrm{Bi}$ cubic interpolation is used in order to obtain smooth surface. A growth factor of ' 2 ' for Bi cubic interpolation is enforced to high frequency sub band images. Stationary wavelet transform (SWT) is adapted to negotiate the information loss in the Sub bands due to down sampling. The MRI Brain tumor image disintegrates into different sub band images by using Stationary wavelet transform, Low-low (LL)), low-high (LH), high-low (HL), and high-high (HH). The SWT High frequency sub bands and interpolated high frequency sub bands are combined each other. Further intensification is achieved by interpolating the estimated High frequency sub bands. The authentic high resolution input image is having admirable information than low frequency sub bands. Hence the interpolation is again applied on the input MRI image Low frequency sub bands.

\subsection{Proposed OHC Scheme}

The proposed Algorithm substantiates the implication of MRI Brain tumor image segmentation and clustering to estimate the tumor area. The proposed approach endorsed optimized results.

\section{Algorithm1:}

Input image $=\mathrm{I}$

Output image $=\mathrm{O}$

Step1. Select and read the input metastasis brain image 'I'

Step2. Reshape 'I' into data sets of column vector $\left(C_{v}\right)$ for segmentation.

Step3. Determine the number of clusters (K) i.e. centroids.

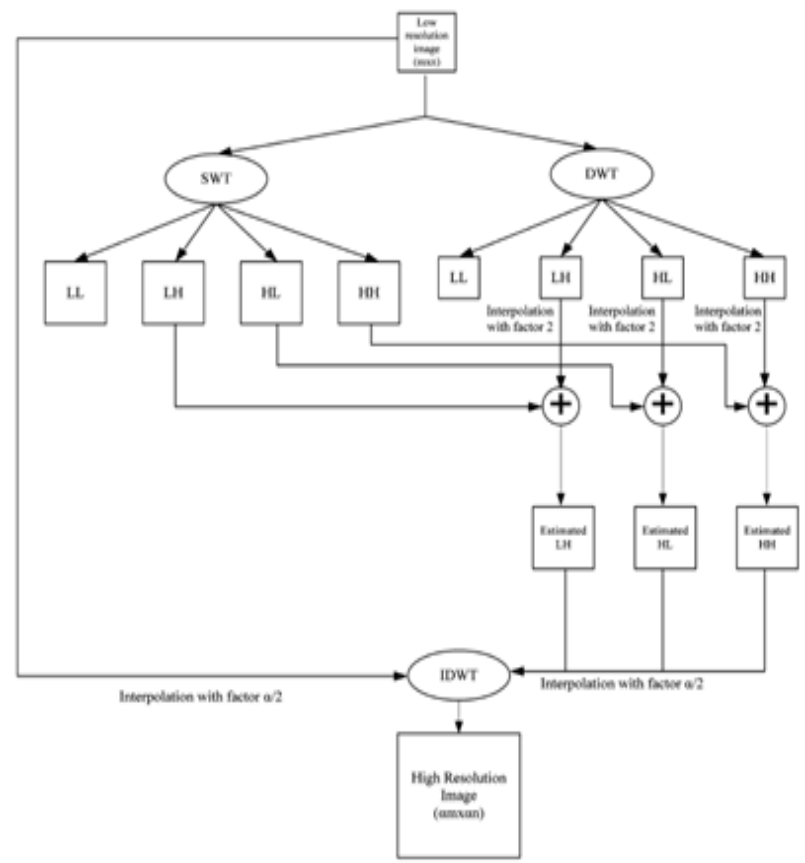

Fig.1 Block Diagram of Super resolution Image enhancement

Step4. Calculate the distance (D) between $C_{v}$ and ' $\mathrm{K}$ ' for each pixel to cluster point

Step5. Find number of $C_{v}$ which are neighboring to the ' $\mathrm{K}$ '.

Step6. Select the ' $\mathrm{K}$ ' with minimum distance and then move 
the $C_{v}$ to the closest relevant centroids

Step8. Re estimate the centroids by selecting the maximum pixel value from the set of relevant centroid data points.

Step9. Repeat the process until the new centroids and the previous centroids are symmetrical.

\section{Algorithm 2: \\ Input image $=\mathrm{O}$ \\ Output image $=\mathrm{S}$}

Step1: Read the output image ' $O$ ', which has been obtained from algorithm1

\section{Step2: Apply Fuzzy C-means algorithm to the image ' $O$ '}

Step3: Display the segmented metastasis brain image in which we had multiple tumors

Step4: To calculate the tumor areas individually, first divide the segmented image by using Split and Merge (SM) algorithm (described in below section)

Step5: After separating the tumors, calculate the area of the tumors by using approximate reasoning method, in which the area of the tumor will be calculated by considering the number of white pixels

Step6: And also compute the CPU time in seconds for the comparison of proposed and existing techniques

\subsection{Split and Merge algorithm}

For estimating the tumor area, the image should be portioned into regions. A set of pixels associated with analogous properties is labeled as a region. Each region is a segment analogous to the input image. Based on the similarity and spatial proximity the pixels corresponding to region are grouped together. Grey value differences and grey value variance is used to achieve similarity. Euclidian distance and compactness of a region is used to achieve spatial proximity. Region splitting and merging approach is used to divide the multiple tumor MRI image into segments. 256*256 MRI image is considered as a single region. The similarity condition is to be tested to split the single region image into subsidiary regions. Quardtree method is adopted for splitting the MRI image. 256*256 MRI image is divided into four $128 * 128$ sub regions, each $128 * 128$ region is further split into four $64 * 64$ sub regions. This process continues until the region fulfills the similarity (homogeneous) condition. The homogeneous two adjacent regions are merged as a single one. This merging process continues until no two regions fulfill homogeneous condition. Since the multiple tumors located at different portions of the image, Split and merge approach is adopted to segment the MRI image. Algorithm1 and 2 is applied to estimate the area of the tumor. And the estimated value is compared with the existing pillar K-Means, K-Means, Fuzzy C-Means, methods.

\subsection{Approximate Reasoning}

Linearization method is adopted to estimate the area of the tumor. The MRI image comprises of two pixel values either gray or white i.e., 0 or 1 . Where ' 0 ' is for black and ' 1 ' is for white pixel value representation. The size of the image is considered to be $256 \times 256$.

$I=\sum_{x=0}^{255} \sum_{y=0}^{255}[f(0)+f(1)]$

Pixels $=$ Width $*$ Height $=256 * 256$

\author{
$\mathrm{f}(0)=$ black pixel ' 0 ' \\ $\mathrm{f}(1)=$ White pixel ' 1 ' \\ Total White Pixels $\left(W_{p}\right)=\sum_{x=0}^{255} \sum_{y=o}^{255} f(1)$
}

$W_{p}=$ number of white pixels

1 Pixel width $=0.264 \mathrm{~mm}$

The equation to estimate the tumor area is

Tumor Size $(S)=\left(\sqrt{W_{p}} * 0.264\right)$

\section{RESULTS AND ANALYSIS}

The multiple tumors were located asymmetrically in the region of the brain. Estimating the area of the single tumor is not a complex task. But multiple tumors at different locations are a great challenge to the physician. The metastasis MRI image may consist of low resolution pixel levels. The combination of DWT and SWT augmenting with the bi-cubic interpolation is enforced to enhance the de-noised tumor image. The major loss of an MRI image after being resolution enhanced by applying interpolation is on its high frequency components, which is by virtue of the smoothing caused by interpolation consequently to increase the quality of the enhanced image. Preserving the edges is imperative. By considering fig 2(a), fig 3(a) and fig4 (a) introducing the SWT information loss due to down sampling in DWT considerably reduced. DWT has been selected to preserve the high frequency components of the MRI image. The LMSE for sample 2 and sample 4 lies within the value "1" and for sample 1 and sample 3 the LSME shows "1.2". The small value of LMSE results fine quality. All "MD" values for all the samples lies Less than ' 1 ' that shows the quality parameters for the resolute images exhibits good quality. The image size of $256 * 256$ is used for enhancement with an interpolation factor of ' 2 '. The resolute image is considered for testing using Region based split and merge approaches. Split and merge approach is tested on MR image Samples. The Fig 3 (b), (c), (d), (e), (f) and (g) represents the experimental results of the sample '1'. The Resolute MR image is considered as a single region and consists of three tumors. The image is split into four sub regions. Region 1 consists of single tumor; whereas region 2 consist of two tumors. Hence region 2 is again split into 2 sub regions. The area of the tumor 1,2, 3 is estimated using the existing segmentation and clustering approaches and also with the proposed method i.e. optimized hybrid clustering algorithm. For sample $1 \mathrm{~K}$-Means and FCM methods produced approximately similar results. Whereas the proposed method exhibits optimum results for tumor 1,2 , and 3 is tabulated with $3.5222 \mathrm{~mm}^{2}, 2.8434 \mathrm{~mm} 2$, and tumor $32.6135 \mathrm{~mm}^{2}$ respectively. The estimated tumor area will be favorable to the Physician for both therapeutic and surgical applications. The proposed approach is handful support to the physician with no damage to the healthy tissue while treating the tumor region. Considering sample 2 and sample 3, the K-means and FCM approaches grouped neighborhood non tumor pixel values into tumor cluster due to grey level similarity. The FCM approach results significantly weaker results. FCM is more depending on the initial centroids of clusters and difficult to determine the membership functions. Hence the results are significantly higher than the proposed method. 


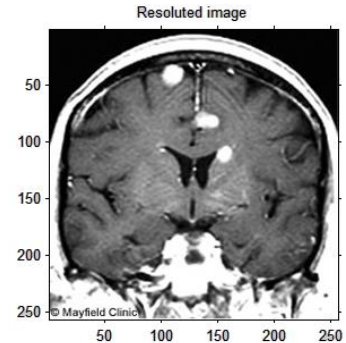

(a)

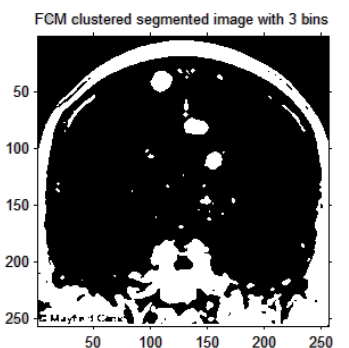

(b)
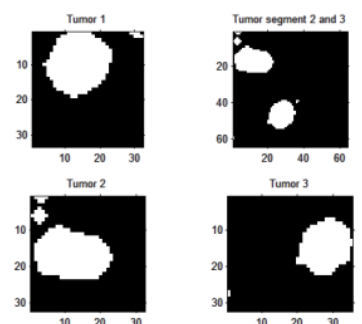

(c)
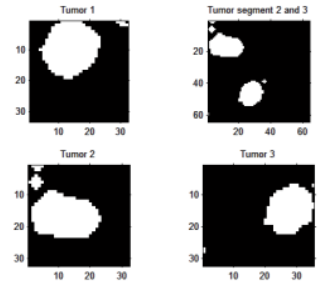

(e)
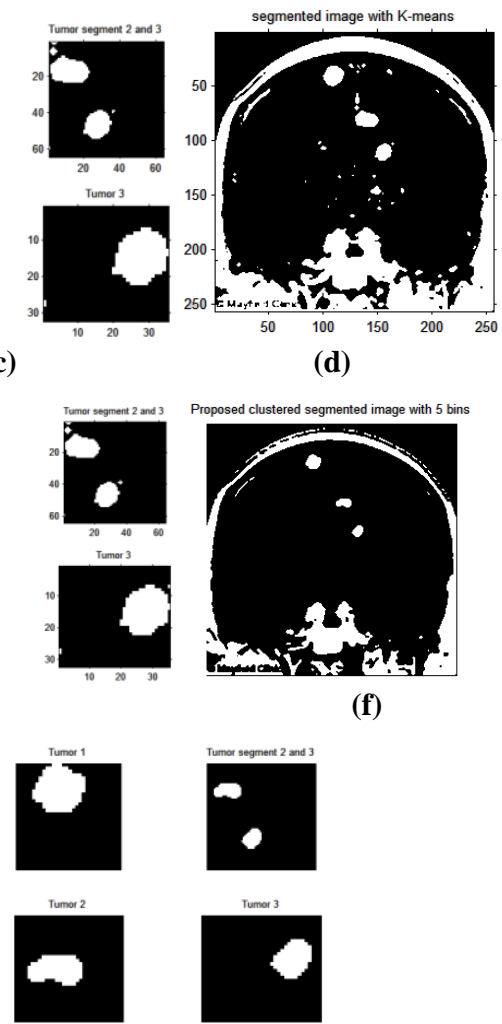

(d)

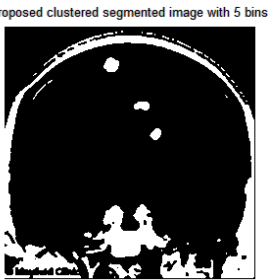

(f)
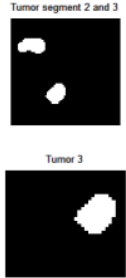

(g)

Fig2 (a) Resolute image (b) Segmented image using FCM (c) Tumors separation using SM for FCM (d)

Segmentation using K-Means (e) separation of tumors using SM for k-means (f) Segmentation using Proposed OHC and (g) SM for proposed

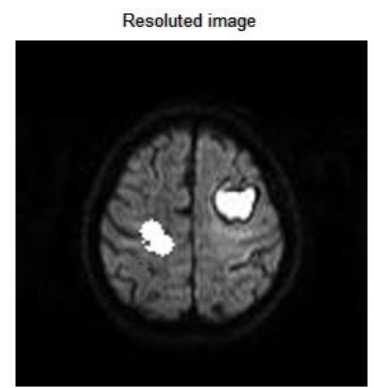

(a)

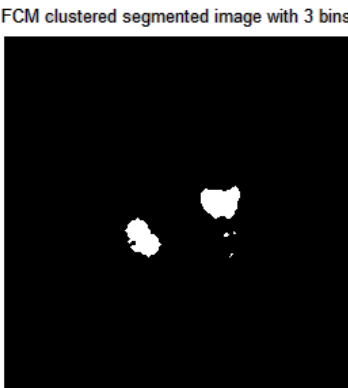

(b)

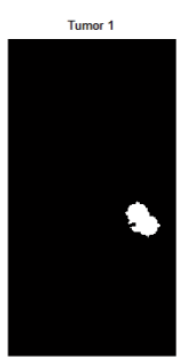

Tumor 1

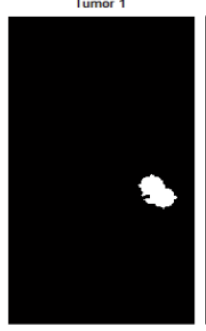

(e)

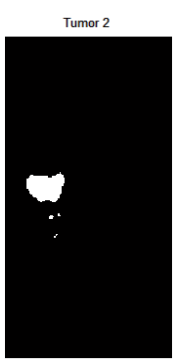

(c)
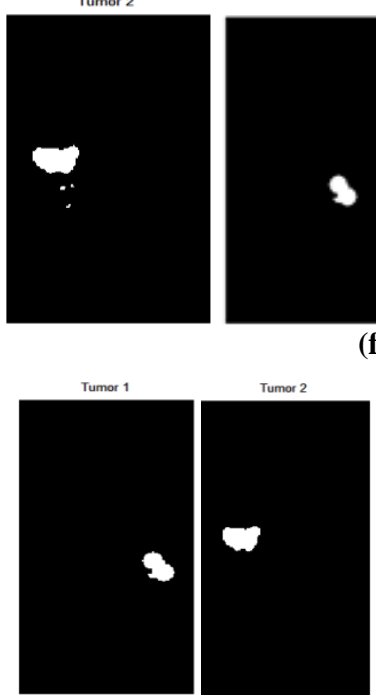

(g)

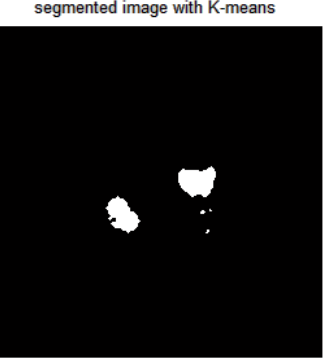

(d)

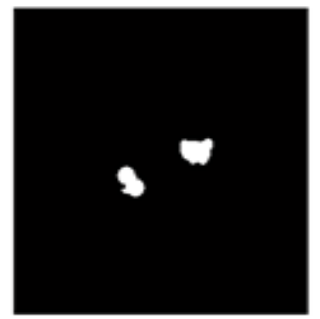

(f)
Fig3 (a) Resoluted image (b) Segmented image using FCM

(c) Tumors separation using SM for FCM (d)

Segmentation using K-Means (e) separation of tumors using SM for k-means (f) Segmentation using Proposed $\mathrm{OHC}$ and (g) SM for proposed

Table1. Comparison of Tumor Areas for Various methods

\begin{tabular}{|l|c|c|c|}
\hline \multirow{2}{*}{ Tumor No } & \multicolumn{3}{|c|}{ Area of the tumor( mm^2 ) } \\
\cline { 2 - 4 } & FCM & K-Means & Proposed \\
\hline Tumor 1 & 4.3540 & 4.3700 & 3.5222 \\
\hline Tumor 2 & 4.2976 & 4.3057 & 2.8434 \\
\hline Tumor 3 & 3.5518 & 3.5811 & 2.6135 \\
\hline \multicolumn{4}{|c|}{ Sample 2 Sample 3 } \\
\hline Tumor 1 & 5.5251 & 5.5251 & 5.0987 \\
\hline Tumor 2 & 6.0259 & 6.0375 & 5.6990 \\
\hline \multicolumn{4}{|c|}{ Sample 4 } \\
\hline Tumor 1 & 11.7591 & 11.8330 & 6.2641 \\
\hline Tumor 2 & 5.6990 & 5.7416 & 3.1899 \\
\hline \multicolumn{5}{|c|}{11.0439} & 10.9520 & 10.4639 \\
\hline Tumor 1 & 8.1327 & 8.1156 & 7.8181 \\
\hline Tumor 2 & \multicolumn{5}{|c|}{} \\
\hline
\end{tabular}




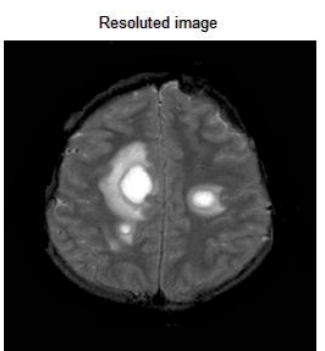

(a)
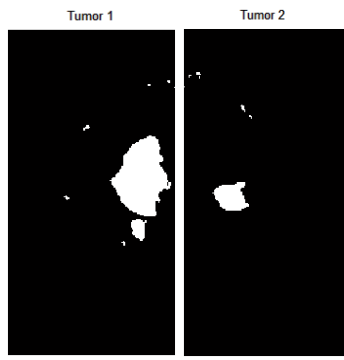

(c)

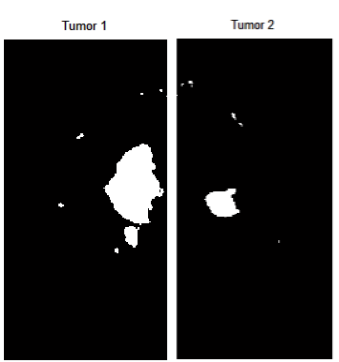

(e)

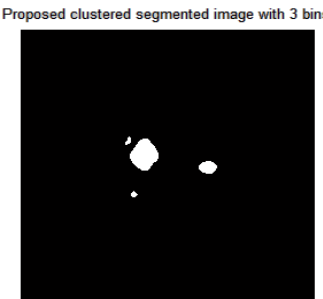

(f)

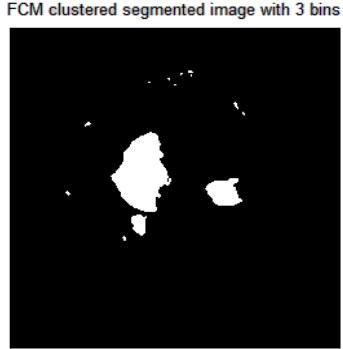

(b)

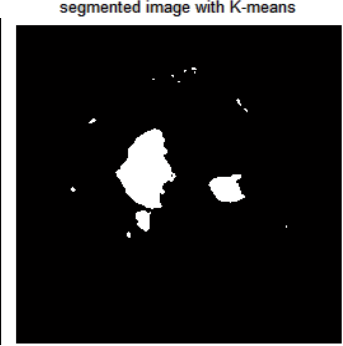

(d)

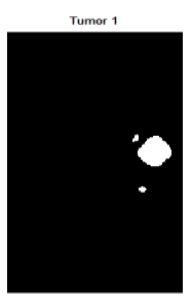

(g)

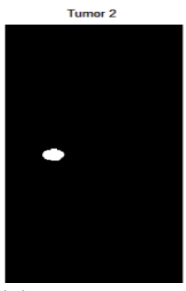

)
Fig4 (a) Resoluted image (b) Segmented image using FCM (c) Tumors separation using SM for FCM (d) Segmentation

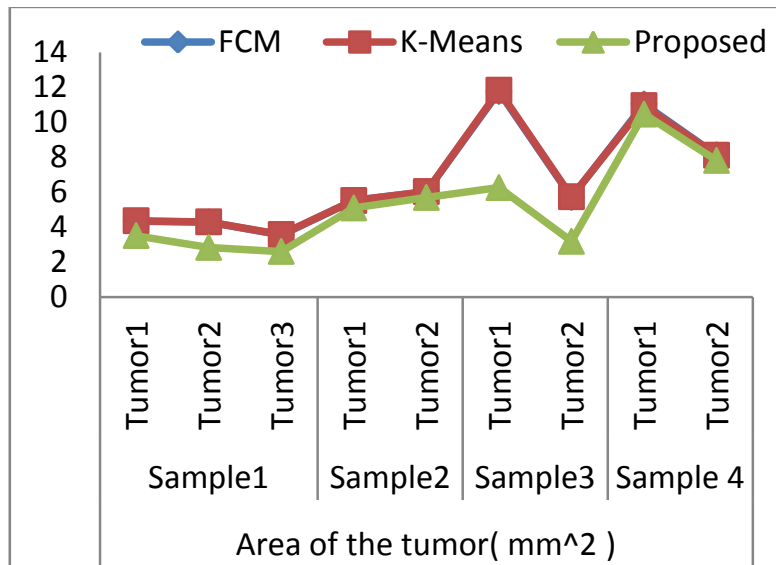

Fig 5. Comparison of Area of the tumors with various approaches
Table2. Quality Analysis Comparison for Various Sample Resolute Images

\begin{tabular}{|l|l|l|l|l|}
\hline $\begin{array}{l}\text { Quality } \\
\text { parameter } \\
\text { s }\end{array}$ & Sample 1 & Sample2 & $\begin{array}{l}\text { Sample } \\
3\end{array}$ & $\begin{array}{l}\text { Sample } \\
4\end{array}$ \\
\hline PSNR(db) & 58.4795 & 67.9731 & 66.0692 & 64.7594 \\
\hline AD & 0.225881 & 0.045542 & 0.07362 & 0.09110 \\
\hline SC & 3.996330 & 3.906995 & 3.90690 & 3.90684 \\
\hline NCC & 0.475903 & 0.495715 & 0.49895 & 0.49853 \\
\hline MD & 0.500000 & 0.000000 & 0.00196 & 0.00000 \\
\hline LMSE & 1.220969 & 1.066670 & 1.21422 & 1.09528 \\
& & & & \\
\hline NAE & 0.000000 & 0.000000 & 0.00000 & 0.00000 \\
& & & & \\
\hline
\end{tabular}

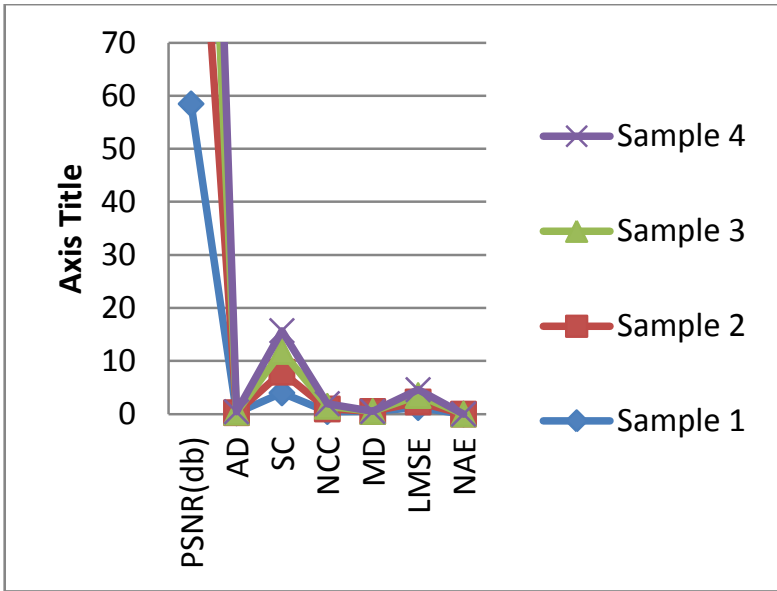

Fig 6. Comparison of Quality parameters for resolute sample images

Table 5. Comparison of Tumor Cells with its Radius and

\begin{tabular}{|c|c|c|c|c|}
\hline $\begin{array}{c}\text { Tumor } \\
\text { No }\end{array}$ & $\begin{array}{c}\text { Radius } \\
(\mathrm{cm})\end{array}$ & $\begin{array}{c}\text { Volume } \\
\left(\mathrm{m}^{3}\right)\end{array}$ & $\begin{array}{c}\text { Number } \\
\text { of tumor } \\
\text { cells } \\
(\mathrm{N}) * \\
10^{11}\end{array}$ & $\begin{array}{c}\text { Uni- } \\
\text { dimensional } \\
\text { diameter } \\
(2 \mathrm{r})\end{array}$ \\
\hline \multicolumn{5}{|c|}{ Sample 1} \\
\hline Tumor 1 & 0.10590 & 4.9732 & 21.18 & 0.2118 \\
\hline Tumor 2 & 0.03030 & 0.11652 & 6.06 & 0.0606 \\
\hline Tumor 3 & 0.02905 & 0.3533 & 5.81 & 0.0581 \\
\hline \multicolumn{5}{|c|}{ Sample 2 } \\
\hline Tumor 1 & 0.0405 & 0.2797 & 8.116 & 0.08116 \\
\hline
\end{tabular}




\begin{tabular}{|c|c|c|c|c|}
\hline Tumor 2 & 0.04290 & 0.07897 & 8.58 & 0.0858 \\
\hline \multicolumn{5}{|c|}{ Sample3 } \\
\hline Tumor 1 & 0.0449 & 0.38099 & 8.98 & 0.0898 \\
\hline Tumor 2 & 0.0320 & 1.3846 & 6.410 & 0.0641 \\
\hline \multicolumn{5}{|c|}{ Sample 4 } \\
\hline Tumor1 & 0.0581 & 0.8226 & 11.62 & 0.1162 \\
\hline Tumor2 & 0.0502 & 0.5312 & 10.05 & 0.1005 \\
\hline
\end{tabular}

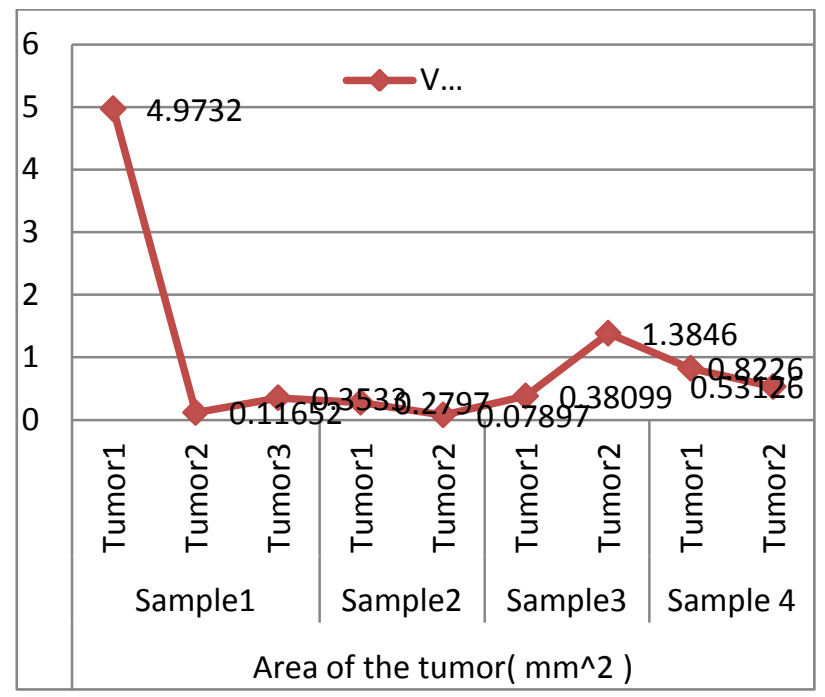

Fig 7. Volume of the tumor for various samples

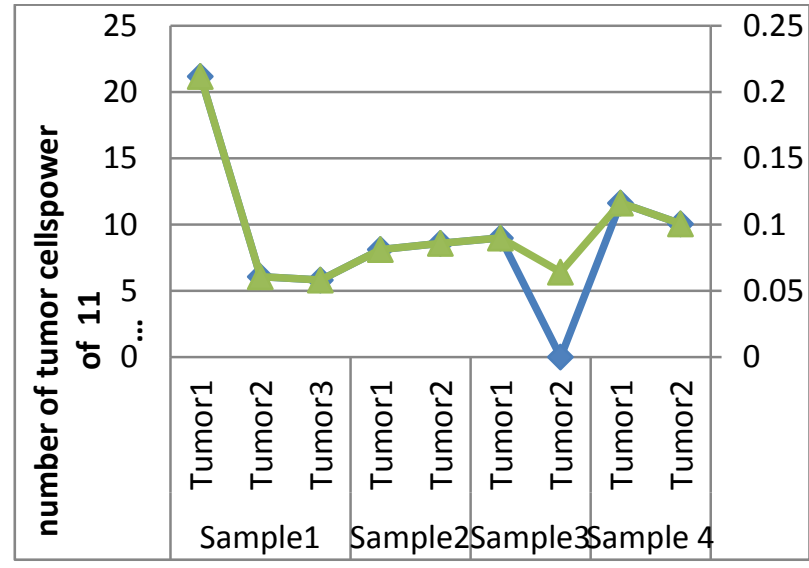

Fig 8. The Number of Tumor cells Against Unidimentional Diameter

\section{CONCLUSION}

The experiments were conducted on metastasis multiple tumor images. The tumors are located at various locations. Split and merge approach is favorably considered for estimating the tumor area. The combination of DWT-SWT in conjunction with bi-cubic interpolation approach results intensified low contrast pixel levels image for better analysis. The valued information at the edges are safeguarded and intensified while evaluating the tumor size. The "Optimized hybrid Clustering Algorithm "proved as a refined and respectable method to estimate the actual area of the tumor. In future the location of the tumor may be determined in addition with the size i.e. area of the tumor. The developed algorithm has been tested on 20 numbers of Metastasis Tumor MR Images. The split and merge mechanism applied on Optimized hybrid clustering algorithm exhibits some limitation to the MRI samples where a tumor lies between the two segments. Some portion of the tumor lies in one segment and the rest of the tumor portion lies in another segment. Even though, this method proved itself as a refined method for metastasis Brain tumor images. The Number of aberrant cell has been quantified by considering one centimeter uni dimensional diameter tumor contains $10^{9}$ tumor cells. Estimating the area and Volume and number of the tumor cells will support for better therapy.

\section{ACKNOWLEDGEMENT}

I thank to Dr.Krupasagar Kalapala and Dr Srujana Ulavapati for their constant support. And I thank to the principal , management of Sai Spurthi Institute of technology.

\section{REFERENCES}

[1] Yu-Hsiang Wang," tutorial on Image segmentation", National Taiwan University.

[2] B.naresh Kumar “ M.Sailaja " An Automated 3D Segmented and DWT Enhanced Model for Brain MRI", International Journal of scientific \& Engineering Research, Vol.3, 2012.

[3] Samir kumar Bandopadyaya " Image Enhancement Technique applied to low-Field MR Brain Images", International Journal of Computer Applications, Vol. 15, feb 2011

[4] Sunaya U.Shirodkar," Image Resolution Enhancement using various wavelet Transfers", International journal of advances in science Engineering and Technology, Vol.1, 2014.

[5] V.C.MIAINDARGI, A.P.MANE "Decimated and UnDecimated Wavelet Transforms based Image Enhancement", International journal of industrial Electrical, Electronics, Control and Robotics, Vol.03,Issue.05,2013

[6] B.Sivakumar, S.Nagaraj "Discrete and stationary wavelet decomposition for image Resolution Enhancement" International Journal of Engineering trends and Technology, Vol. 4,2013

[7] Mr.G.M.Khaire, R.P.Shelkikar,” Resolution Enhancement of images with interpolation and DWT SWT Wavelet domain components", International Journal of Application or Innovation in Engineering and Management, Vol.2, 2013.

[8] Manisha Bhagwatl, R.K.Krishna\& V.E.Pise, "Image Segmentation by Improved Watershed Transformation in Programming Environment MATLAB" International Journal of Computer Science \& Communication Vol. 1, No. 2, pp. 171-174, 2010.

[9] M.H. Fazel Zarandia, M. Zarinbala, M. Izadi, "Systematic image processing for diagnosing brain tumors: A Type-II fuzzy expert system approach," Applied soft computing, pp: 285-294, 2011

[10] S. Zulaikha BeeviM, Mohamed Sathik, "An Effective Approach for Segmentation of MRI Images: Combining Spatial Information with Fuzzy C-Means Clustering" European Journal of Scientific Research, Vol. 41, No.3, pp.437-451, 2010. 
[11] S. Mary Praveena, Dr.I1aVennila, "Optimization Fusion Approach for Image Segmentation Using K-Means Algorithm" International Journal of Computer Applications, Vol 2, No.7, June 2010.

[12] M. Masroor Ahmed \& Dzulkifli Bin Mohammad, "Segmentation of Brain MR Images for Tumor Extraction by Combining K-means Clustering and Perona-Malik Anisotropic Diffusion Model" International Journal of Image Processing, Vol. 2, No. 1, 2010

[13] Tse-Wei Chen, Yi-Ling Chen, Shao-Yi Chien, "Fast Image Segmentation Based on K-Means Clustering with Histograms in HSV Color Space" Journal of Scientific Research, Vol. 44 No.2, pp.337-351, 2010.

[14] Anil Z Chitade, " Colour based image segmentation using k-means clustering" International Journal of Engineering Science and Technology Vol. 2(10), 53195325,2010

[15] Selvakumar, J., Lakshmi, A., Arivoli, T., "Brain Tumor segmentation and its area Calculation in Brain MR images using K-means Clustering and Fuzzy C-means algorithm", International Conference on Advances in Engineering, Science and Management (ICAESM), pp: 186-190, 2012

[16] Barakbah, A.R., Kiyoki. Y., "A Pillar algorithm for $K$ means Optimization by Distance Maximization for Initial Centroid Designation", IEEE Symposium on Computational Intelligence and Data Mining, pp: 61-68, 2009.
[17] K. Vidyasagar, Dr. A. Bhujangarao, Dr. T. Madhu," Brain tumor detection and its area estimation using Pillar K-Means Algorithm", International journal of Engineering sciences research, Vol. 05,march 2014

\section{AUTHOR PROFILE}

K. Vidyasagar Received the B.Tech degree in Instrument Technology from Andhra University College of Engineering Visakhapatnam, the M.E from P.S.G.Tech Coimbator. He is now a research scholar under the guidance of Dr.A. Bhujangarao,Andhra University. His current research interests include image processing in biomedical instrumentation and related embedded systems

Dr. A. Bhujangarao received Ph.D in Instrumentation from Andhra University. who is currently professor and HOD, at Department of Instrument Technology Andhra University college of Engineering, Visakhapatnam, he is specialized in Instrumentation and acoustic remote sensing Instrumentation, he published 30 journal papers and 23 conference papers. His current research interest is on Bio Medical instrumentation and process control instrumentation.

Dr. T. Madhu received Ph.D., from Osmania University, Hyderabad. He is now working as Principal, Swarnandhra Institute of Engineering \& Technology, Seetharampuram, Narasapur. His current research interests include GPS data analysis \& Modeling, speech \& image processing and RF design.able, try the font named Computer Modern Roman. On a Macintosh, use the font named Times. Right margins should be justified, not ragged. 